\title{
How robust is a LoRa communication against impulsive noise?
}

\author{
$1^{\text {st }}$ Jules Courjault \\ $2^{\text {nd }}$ Baptiste Vrigneau \\ $3^{\text {rd }}$ Olivier Berder \\ $4^{\text {th }}$ Manav R. Bhatnagar \\ Univ Rennes, CNRS, IRISA \\ Univ Rennes, CNRS, IRISA \\ Univ Rennes, CNRS, IRISA \\ jules.courjault@irisa.fr \\ Lannion, France \\ Lannion, France \\ baptiste.vrigneau@irisa.fr \\ olivier.berder@irisa.fr \\ Department of Electrical Engineering \\ Indian Institute of Technology Delhi, \\ New Delhi, India \\ manav@ee.iitd.ac.in
}

\begin{abstract}
In the last decade Internet of Things (IoT) grew up in an exponential behavior with applications requiring long range and low power wireless transmissions. Factory of the Future (FoF), also called Industry 4.0, aims to use IoT technologies to enhance productivity, therefore adding high reliability constraints. Several IoT standards were proposed and LoRa has emerged as a high potential candidate for a variety of applications. LoRa modulation is based on a chirp spread-spectrum technique and offers efficient transmission up to 50 kbps over several kilometers. The performance of LoRa in terms of symbol or bit error probability has been theoretically analyzed in few recent papers for a Gaussian channel. However, the industrial environment is often corrupted with impulsive non Gaussian noise generated by high-power equipment. In this paper, the impact of impulsive noise, modeled by the Middleton Class-A noise, is introduced and the robustness of a LoRa communication is studied. Compared to the Gaussian case, simulations show that impulsive noise may severely degrade system performance. This Signal-to-Noise Ratio loss can reach up to $10 \mathrm{~dB}$, but increasing the spreading factor can reduce the noise impact.
\end{abstract}

Index Terms-IIoT, LoRa performance, Symbol error rate, impulsive noise, Middleton Class A

\section{INTRODUCTION}

During the last decade, the number of small connected nodes, denoted Internet of Things (IoT), has grown up exponentially and are used for various applications. As an example, connected farms [1] aim to help farmers to survey their agricultural infrastructures. In another context, Factory of the future $(\mathrm{FoF})$ or Industry 4.0 is considered as the fourth industrial revolution. Artificial intelligence may help the optimization of the production, but it generally requires huge amount of data and thus a very efficient way to collect the information. Wireless sensors are the first step of this process and must tackle constrained challenges concerning the energy consumption, long range, high reliability and security. Since several years, wireless transmission for IoT has been studied [2] in order to enhance the distance range and the energy efficiency. If $5 \mathrm{G}$ is a natural candidate [3], specific IoT standards already in place as LoRa [4] [5] and Turbo-FSK [6] may also be applied to the Industrial IoT (IIoT) [7]-[9].

A factory represents however a very specific environment that can vary a lot from one place to another. Indeed, the indoor environment with a lot of metallic structures and multiple reflectors may lead to severe attenuation, and specific power equipment or machine tools generate noise. The main issue is that noise cannot be considered as Gaussian any more and has an impulsive behavior. This can lead to a performance degradation of the wireless link and impact the energy consumption and the reliability, crucial to the FoF. For example, a high voltage substation uses a lot of power devices and the monitoring is disturbed with an impulsive noise modeled with a Middleton distribution [10], [11]. In order to efficiently deploy wireless sensor nodes in factories, there is a crucial need for fast and accurate performance estimation of IoT technologies in this particular context. This paper aims to fill this gap and evaluate the robustness of LoRa technology under severe propagation conditions induced by impulsive noise.

The rest of the paper is organized as follows. Section II gives details about the IoT standards and impulsive noise distribution. Section III focuses on LoRa transmission and existing performance evaluation methods. Section IV presents simulation results and discussions about the impact of noise impulsiveness on performance in term of symbol error rate (SER) and some conclusions are drawn in Section V.

\section{FACTORY OF THE FUtURE ENVIRONMENT}

FoF is considered as a severe and difficult environment in terms of fading but also of noise. This section introduces the impulsive noise, the existing models and also presents IIoT technologies focusing on the LoRa standard.

\section{A. Impulsive noise modeling}

Propagation models for FoF environment, where signals usually suffer severe degradation, can be organized into two groups, namely semi-probabilistic and semi-deterministic. The proposed study will not consider the fading issue but mainly focuses on noise, which can be very specific in such a context. As introduced in [10], [11], the noise is indeed more impulsive than Gaussian, leading to a degradation of performance in terms of reliability and energy consumption.

In the literature, different models can be found for impulsive noise, e.g. Middleton noise [12], generalized Gaussian [13], $\alpha$-stable [14]. In this paper we choose to focus on Middleton noise, in particular the Middleton Class-A Noise (MCAN). Middleton noise models are indeed divided in 3 classes: Class A with a noise band narrower than the receiver bandwidth, 


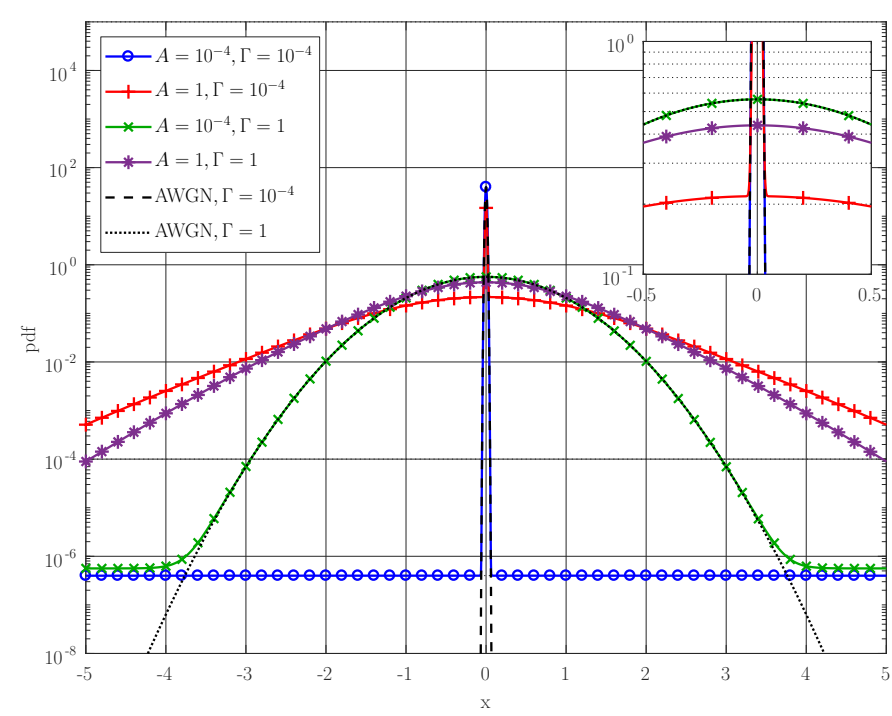

Fig. 1. Theoretical PDFs of MCAN for different parameters $\Gamma$ and $A$.

Class $\mathrm{B}$ with a noise band as broadband for the receiver, and Class $\mathrm{C}$ as the sum of Class A and Class B. For the MCAN the probability density function (PDF) is defined as [12]:

$$
f(x)=\sum_{m=0}^{+\infty} \frac{A^{m} e^{-A}}{m !} \frac{1}{\sqrt{2 \pi \sigma_{m}^{2}}} e^{-\frac{x^{2}}{2 \sigma_{m}^{2}}},
$$

where $\sigma_{m}^{2}$ is equal to $\left(\frac{m}{A}+\Gamma\right) \sigma^{2} /(1+\Gamma), A$ is the impulse index equal to $\eta \tau / T_{0}$ with $\eta$ representing the number of pulses during $T_{0}$, and $\tau$ denotes the average duration of a pulse. $\Gamma=$ $\sigma_{g}^{2} / \sigma_{i}^{2}$ is the impulsive to background ratio, and $\sigma^{2}=\sigma_{g}^{2}+\sigma_{i}^{2}$ is the background plus impulsive power. The Middleton noise is therefore characterized by 3 parameters:

- $A$ sets the impulsiveness of the noise and represents the density of pulses in an observation period [15]. It is a positive real value inferior to 1 .

- $\Gamma$ is defined as the power ratio between Gaussian and impulsive parts. A small value of $\Gamma$ means that impulsive values appear with a high power.

- $\sigma^{2}$ is the total noise power.

Fig. 1 compares the PDFs of MCAN for different sets of parameters with the one of Additive White Gaussian Noise (AWGN). The variance of the AWGN has been adjusted to correspond to the background power of MCAN, i.e. $\sigma_{A W G N}^{2}=$ $\Gamma /(1+\Gamma) \sigma_{M C A N}^{2}$. The Middleton noise may have a heavy tail compared to Gaussian distribution. As we can see from the figure, when $A$ increases, the MCAN tends toward an AWGN. In the case of high value of $A$ (near 1), the impact of $\Gamma$ is insignificant. For the rest of the paper, we will consider these 4 representative impulsive noise configurations.

\section{B. LoRa for Industrial IoT}

Authors of [16] [2] present a panel of standards for Industrial IoT (IIoT), such as NB-IoT, Sigfox or LoRa. In [8] authors explain that nodes of an industrial network have an update time of 60 seconds or slower. LoRa is a long range, lowpower, and low data rate communication technology promoted by the LoRa Alliance, is already available and very popular. Moreover, in [8] authors study and demonstrate the feasibility of a LoRa network in an industrial application, i.e. the refresh time is slower than 60 seconds. For these reasons, the symbol error rate of LoRa technology in an industrial environment will be studied in this paper.

The LoRa physical layer is developed by Semtech [4] [5]. This communication can use frequency bands of $433 \mathrm{MHz}$, $868 \mathrm{MHz}$ or $915 \mathrm{MHz}$ and its data rate can reach up to $50 \mathrm{kbps}$. It uses the Chirp Spread Spectrum (CSS) modulation with a linear variation of frequency on time [17] [18], which allows the reduction of both interference and Doppler effects [19]. The modulation can be configured with three parameters: the bandwidth $B W(125,250$ or $500 \mathrm{kHz})$, the spreading factor $S F$ (from 7 to 12 ), and the coding ratio $C R$ (4/5 to 4/8). The Figure 2 illustrates the observed spectrum of a LoRa symbol as a function of $S F$.

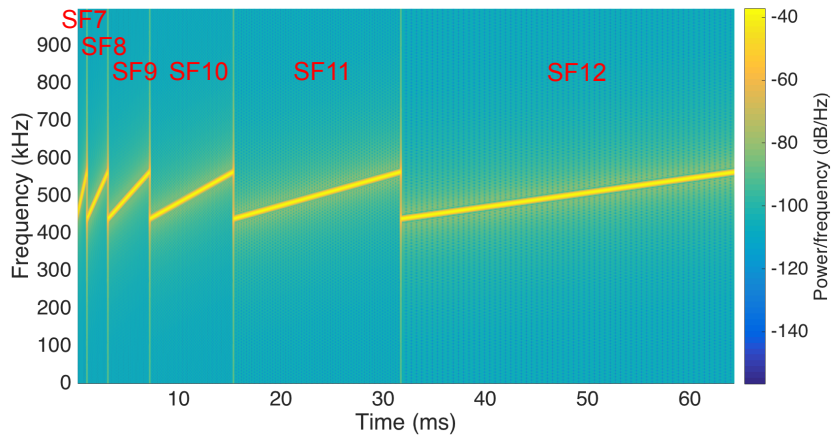

Fig. 2. LoRa transmitted symbols with different values of $S F$.

\section{Performance eValuation of LoRa}

This section introduces in details the LoRa communication in order to identify all the mathematical steps and difficulties to overcome to obtain theoretical SER. Examples for the AWGN case are showed and previous works on communications with impulsive noise are summed up.

\section{A. How does LoRa work?}

In LoRa, the spreading factor, $S F$, is defined as the logarithm in base 2 of the number of chirps per symbol. A LoRa symbol is therefore composed of $2^{S F}$ chirps covering the entire bandwidth, starting with a series of upward (or downward) chirps from an initial frequency, which represents a code word. The frequency will wrap to the minimum frequency (or maximum frequency with down-chirp) when the maximum (minimum with down-chirp) of the bandwidth is reached. A linear chirp signal is usually defined by:

$$
s(t)=e^{j\left(2 \pi\left(f_{0} t+\frac{\mu t^{2}}{2}\right)+\phi_{0}\right)},
$$

where $\phi_{0}$ is the initial phase, $\mu$ the chirp rate and $f_{0}$ the starting frequency of the chirp. $\phi(t)=2 \pi\left(f_{0} t+\frac{\mu t^{2}}{2}\right)+\phi_{0}$ 


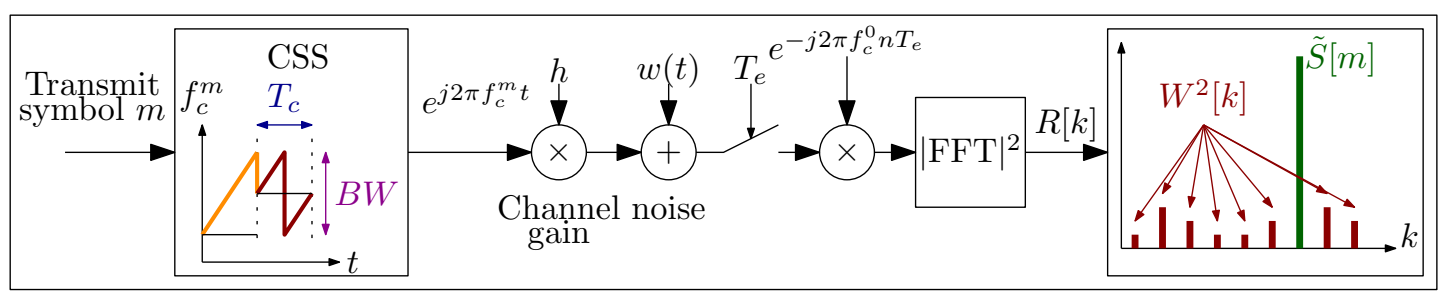

Fig. 3. Bloc diagram of a LoRa transmission: the LoRa symbol is corrupted by an additive noise (channel gain $h=1$ in this study).

is the instantaneous phase at time $t$, and the corresponding instantaneous frequency is:

$$
f(t)=\frac{d \phi(t)}{2 \pi d t}=f_{0}+\mu t .
$$

Applying CSS to LoRa modulation, each frequency $f_{0}$ in the band represents a code word. The frequency is swapped at the maximum value of bandwidth, the instantaneous frequency is therefore represented as a modulo operator of $B W$ :

$$
f(t)=f_{\min }+\left[\left(\Delta f_{0}+\mu t\right) \bmod B W\right],
$$

where $\Delta f_{0}=f_{0}-f_{\min }$ and $f_{\min }$ is the minimum frequency of the bandwidth.

With $2^{S F}$ chirps per symbol, a code word can contain $S F$ information bits. The duration $T_{c}$ of a symbol is given by: $T_{c}=2^{S F} / B W$. For a given bandwidth, increasing the spreading factor by one unit doubles the Time-on-Air (ToA) to transmit the same amount of data, resulting in the decrease of the bit rate:

$$
R_{b}=S F \times \frac{B W}{2^{S F}} .
$$

The third parameter in the configuration of LoRa modulation is the code rate. LoRa can use Forward Error Correction (FEC) code for each block of four information bits. The number of redundant bits for each block varies from one to four, corresponding to Code Rates $(C R)$ of $4 / 5,4 / 6,4 / 7$ and $4 / 8$. Then, the bit stream is processed by an interleaver to make FEC code more robust to burst errors. The transmitted signal is corrupted by an additive noise, thus we consider the folowing model for the received signal $r(t)$ :

$$
r(t)=s(t)+w(t),
$$

with $s(t)$ the emitted signal, and $w(t)$ the additive noise. The received signal is multiplied with the chirp symbol and an FFT is computed. The decision rule is based on the square modulus, i.e. the periodogram, and the theoretical result is a Dirac centered on the transmit symbol subscript corrupted with a noise $(\tilde{S}[m])$. The maximum rule is applied to estimate the symbol. The different steps of the transmission and reception are presented on Fig. 3.

\section{B. Existing approximations for $A W G N$}

Several authors [20]-[22] were able to give the exact expression of the SER for a LoRa communication. However, the derived SER expression cannot be numerically computed due to a convergence issue of a sum with a large number of binomial coefficients with a high value. Another approach based on $Q$ function was proposed in [21] by approximating Rice and Rayleigh laws by a Gaussian one:

$$
\mathrm{SER} \approx Q\left(\frac{\sqrt{N \cdot \mathrm{SNR}}-\left(H_{N-1}^{2}-\frac{\pi^{2}}{12}\right)^{1 / 4}}{\sqrt{H_{N-1}-\sqrt{H_{N-1}^{2}-\frac{\pi^{2}}{12}}+0.5}}\right)
$$

where $H_{m}$ is the $m^{\text {th }}$ harmonic number which can be approximated by $H_{m} \approx \log (m)+\frac{1}{2 m}+0.57722$ and SNR denotes the Signal-to-noise ratio. Another approximation proposed in [22] is based on the limitation of Taylor series of $(1+x)^{N}$ :

$$
\begin{array}{r}
\mathrm{SER} \approx 1+ \\
\frac{1}{N} \sum_{k=1}^{\epsilon+1} C_{N}^{k}(-1)^{k} e^{-\frac{N \cdot \mathrm{SNR}(k-1)}{k}} Q_{1}\left(\sqrt{\frac{2 N}{k} \mathrm{SNR}}, \sqrt{k z_{c}}\right),
\end{array}
$$

where $\epsilon$ is the development order with typical value of 3 or $5, z_{c}$ is a threshold depending on the value of SF, $\epsilon$ (see [22, Eq.(13)] for the expression of $z_{c}$ ), and $Q_{1}$ is the Marcum $-Q$ function of order 1 defined by:

$$
Q_{M}(a, b)=\int_{b}^{\infty} x\left(\frac{x}{a}\right)^{M-1} e^{-\frac{x^{2}+a^{2}}{2}} I_{M-1}(a x) d x .
$$

\section{Previous studies with MCAN}

It was shown in previous works [10], [11], [15], [23] that impulsive noise modeled with Middleton distribution has an important impact when the receiver was designed with the assumption of Gaussian noise. A typical behavior of the SER is the presence of a flat part leading to a significant SNR loss. The immediate consequences are a loss of reliability, an increase of the errors and a higher required transmit power leading to an energy over-consumption. However, the impulsive noise can be fought thanks to an error coding (in fact mostly at decoding with a soft decoder taking into account the Middleton distribution). A LoRa transmission has a FEC and interleaver but they are very simple. The FEC indeed consists in a Hamming code with 4 input bits and the correcting ability is therefore limited at the best to one bit when considering a hard decoding. Recently, in [24], a Bit Interleaved Code Modulation (BICM) was shown to have better performance than hard decoding for AWGN noise, in LoRa communications. As we want to evaluate precisely the impact of impulsive noise on a LoRa transmission, only the uncoded scheme is considered. 


\section{IMPACT OF Middleton Class-A Noise}

\section{A. Impact of impulsive noise on SER}

In order to evaluate the impact of impulsive noise on LoRa transmission and eventually tune LoRa parameters consequently, the SER is simulated for the 4 previously introduced impulsive noises. We used Monte-Carlo simulations for $S F=$ 7 and $S F=12$ with 10000 and 1000 symbols, respectively. For each configuration, the simulation was iterated until we get, at least, 100 errors on symbols.

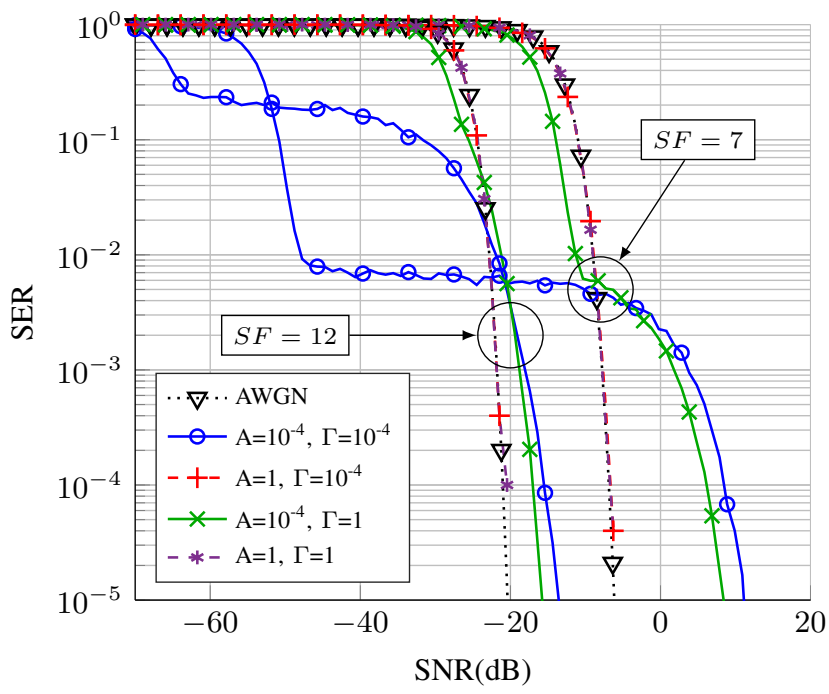

Fig. 4. SER of LoRa communication in MCAN environment for $S F=7$ and $S F=12$, simulated with Monte-Carlo method.

Fig. 4 compares the results of simulations with the theoretical expression (8) of SER for AWGN noise. First, we can observe a flat part as in previous works on impulsive noise for other communications schemes. However, it is obvious that the SNR shift is more significant for low values of $S F$ when the noise is strongly impulsive (i.e. both cases $\left(A=10^{-4}\right.$, $\left.\Gamma=10^{-4}\right)$ and $\left.\left(A=10^{-4}, \Gamma=1\right)\right)$. For the 2 other impulsive cases $\left(A=1, \Gamma=10^{-4}\right)$ and $(A=1, \Gamma=1)$, the SERs are very close to the Gaussian one. At a common target SER $=10^{-4}$, an SNR loss is observed that depends on noise parameters, whose impact will be discussed in the next section.

\section{B. SNR loss for a targeted SER}

The SNR loss, denoted $\triangle \mathrm{SNR}$, corresponds to the difference between the required SNR to obtain a target SER for impulsive noise and for AWGN case. As the Middleton noise has 2 parameters, we vary the value of $\Gamma$ for a given $A$. Fig. 5(a) plots the curves for $A=10^{-2}$ and $S F$ equal to 7 to 12 , and Fig.5(b) for $A=10^{-4}$.

From the figures, we can observe a flat behavior when $\Gamma$ is smaller than $10^{-1}$ and then Middleton case tends toward the AWGN one when $\Gamma$ increases, i.e., $\triangle \mathrm{SNR}$ tends to 0 . Moreover, the SNR loss is decreasing when $S F$ is increasing with the best case for $S F=12$. To confirm this trend, the

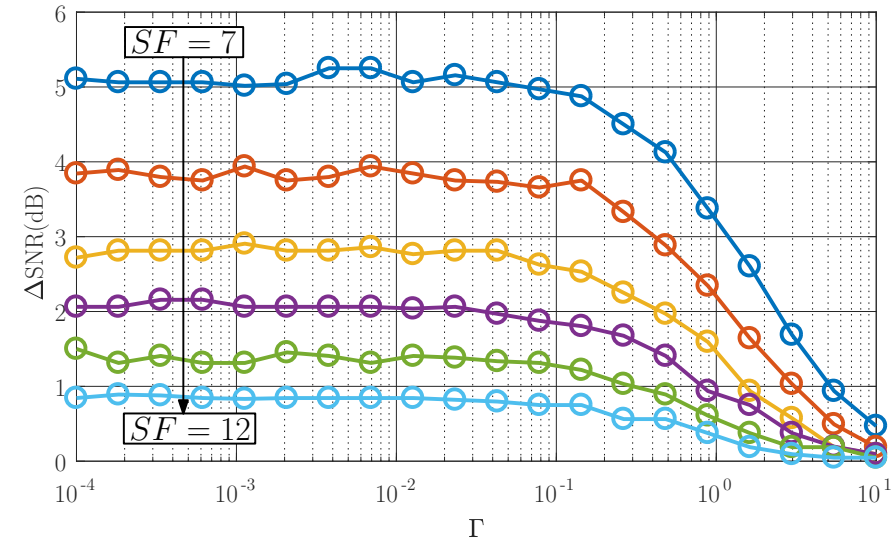

(a) $\triangle \mathrm{SNR}$ for $A=10^{-2}$

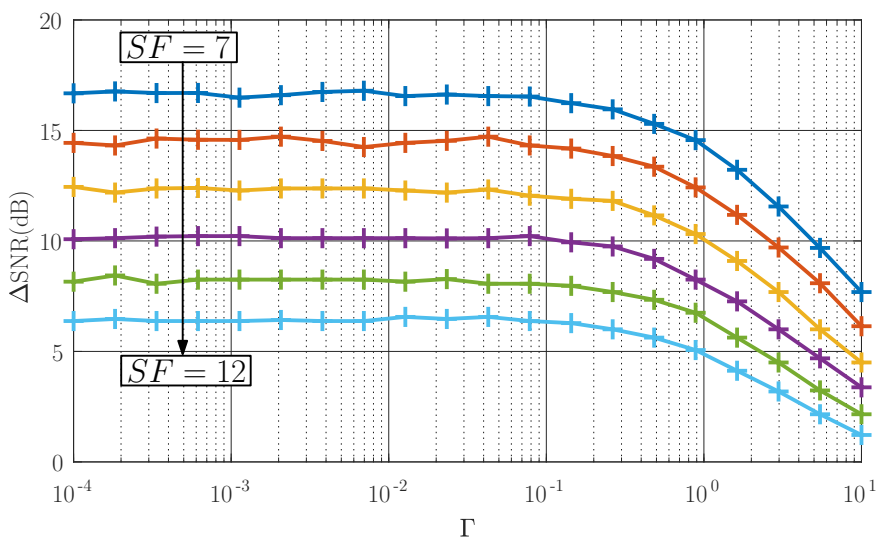

(b) $\triangle \mathrm{SNR}$ for $A=10^{-4}$

Fig. 5. $\Delta \mathrm{SNR}(\mathrm{dB})$ as a function of $\Gamma$, for different values of $A . \Delta \mathrm{SNR}$ is the SNR (dB) gap between AWGN and MCAN to get a SER of $10^{-4}$.

values of $\Delta$ SNR for small values of $\Gamma$ are plotted on Fig. 6 as a function of $S F$. It can be seen from the figure that increasing the value of $S F$ limits the impact of the impulsive noise. In order to be more accurate, Table I shows the value of $\Delta \mathrm{SNR}$ in the flat part $\left(\Gamma<10^{-1}\right)$. As we can see in this table the loss is less important when $A$ increases, moreover it decreases loglinearly with respect to SF. As stated above a strong impulsive noise can degrade the SER and the variation can reach more than $16 \mathrm{~dB}$ for $S F=7$, but tuning SF properly allows to reduce this loss until $6 \mathrm{~dB}$ for $S F=12$. Of course one has to keep in mind that a higher $S F$ induces a longer time-on-air, and a trade-off has then to be found between robustness and energy.

If we look at Fig. 7 representing the impact of $A$ for a given $\Gamma=10^{-4}$, the SNR loss monotonically decreases when $A$ increases until it reaches a Gaussian behavior. But as far as LoRa spreading factor is concerned, it can be concluded again that high values of $S F$ help to overcome the impulsive noise. Increasing $S F$ from 7 to 12 reduces the SNR loss (about $4 \mathrm{~dB}$ and $8 \mathrm{~dB}$ at $A=10^{-2}$ and $A=10^{-4}$, respectively). 


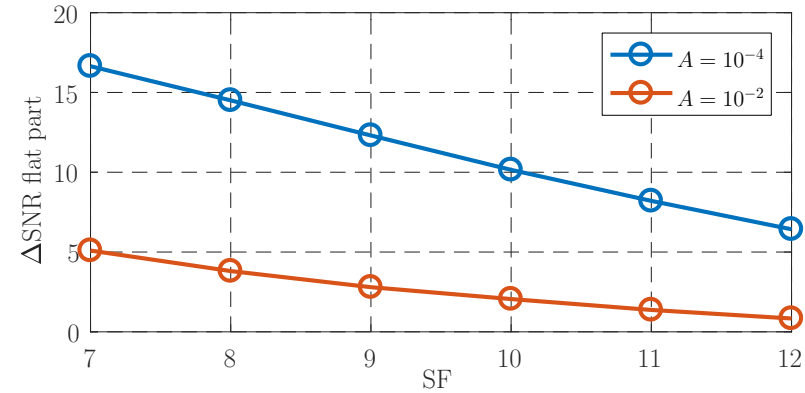

Fig. 6. Values of the flat part of $\triangle \mathrm{SNR}$ for $A=\left\{10^{-4}, 10^{-2}\right\}$ and Gamma $=10^{-2}$

TABLE I

VALUE OF $\Delta$ SNR IN DB IN THE FLAT PART FOR $A=\left\{10^{-2}, 10^{-4}\right\}$ AND $S F$ FROM 7 TO 12

\begin{tabular}{|c|c|c|c|c|c|c|}
\hline $\mathrm{A}$ & $\mathrm{SF}$ & 7 & 9 & 10 & 11 & 12 \\
\hline $10^{-2}$ & 5.09 & 3.81 & 2.80 & 2.05 & 1.37 & 0.84 \\
\hline $10^{-4}$ & 16.64 & 14.50 & 12.30 & 10.15 & 8.21 & 6.43 \\
\hline
\end{tabular}

\section{Robustness of LoRa against impulsive noise}

Fig. 8 shows the MCAN behavior in time domain for different sets of parameters. As can be seen from the figure, the parameter $A$ is linked to the number of pulses in a given interval of time. The choice of $S F$ impacts directly the Time on Air (ToA) of the transmission. For example, Table II reminds the ToA for different values of $S F, B W$ and $C R$ with a payload of 20 bytes. Surprisingly, the strategy based on the minimization of the ToA (leading to a small value of $S F$ ) in order to avoid the high noise values seen in Fig. 8, is not the best. An explanation to this behavior is that the LoRa receiver changes the noise distribution when performing unspreading and FFT operations. Fig. 9 shows the estimated PDF of the noise after those operations and before the square modulus and maximum rule decision. The estimated PDFs for $S F=7$ and for $S F=12$ are shown on Fig. 9(a) and Fig. 9(b), respectively, and both cases use the 4 sets of parameters previously used. The noise is complex but simulations show that the real and imaginary parts are identically distributed. As we can see on Fig. 9 and compared to Fig. 1, both cases with low impulsive noise ([ $\Gamma=1, A=1]$ and $\left.\left[\Gamma=10^{-4}, A=1\right]\right)$ are still close to a Gaussian case, for any value of $S F$. However, when the noise is heavily impulsive, increasing the value of $S F$ leads to mitigating the tail, thus the impulse part of the noise (i.e. the tail) has less impact on the degradation. To sum up, the impulsiveness of the noise is less strong after the FFT when $S F$ increases thanks to the mitigation of the heavy tail by the FFT. An explanation is that the FFT spreads the pulse power over the $N=2^{S F}$ frequency values.

\section{CONCLUSION}

This paper investigated the impact of impulsive noise, on LoRa communication systems, widely used in Industrial IoT. Thanks to simulations, performance in terms of SER and SNR loss was evaluated when the signal is corrupted by a Middleton

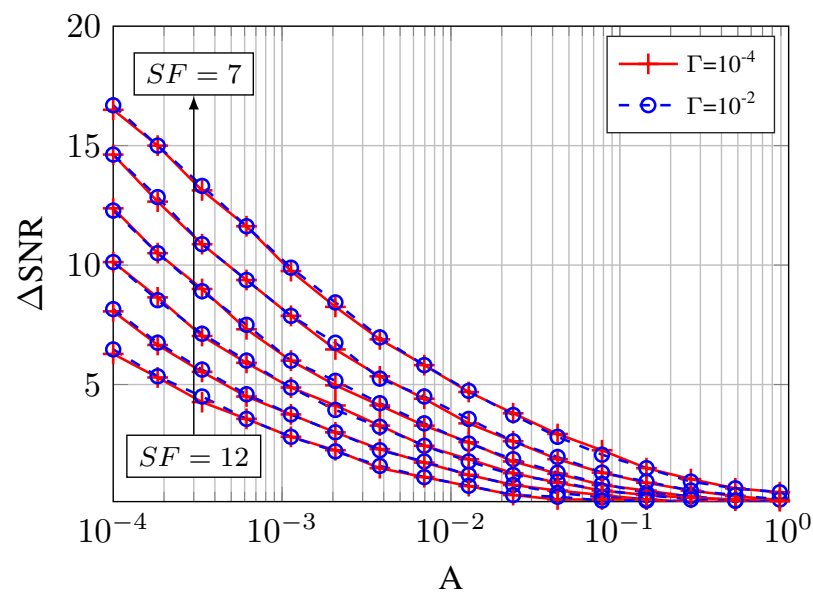

Fig. 7. $\triangle \mathrm{SNR}$ as a function of $A$ for a given $\Gamma=\left\{10^{-2}, 10^{-4}\right\}$ and a target SER $=10^{-4}$

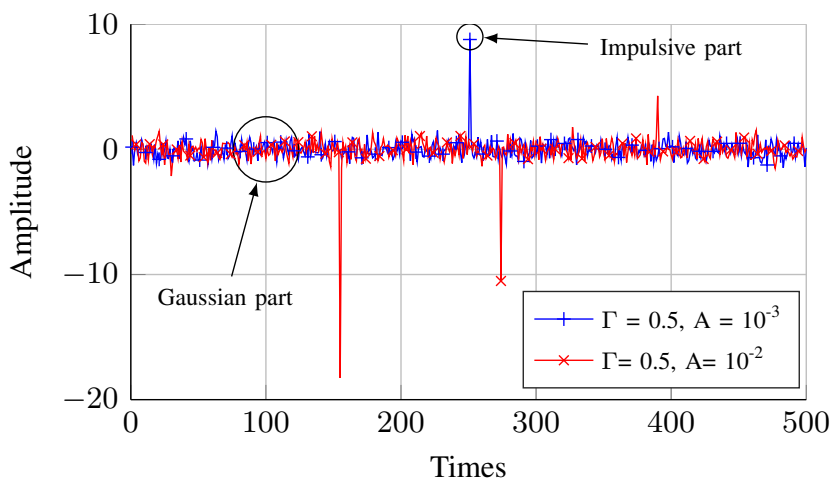

Fig. 8. Middleton noise behavior in time domain for $A=\left\{10^{-3}, 10^{-2}\right\}$ and $\Gamma=0.5$.

noise. Surprisingly, the best strategy is not to minimize the time on air, in order to minimize a significant noise event during the frame transmission, but to increase $S F$ in order to reduce the impulsiveness of the equivalent noise after FFT. This leads of course to larger delay and energy consumption, but on the other hand increasing the spreading factor can drastically reduce the SNR loss (up to $10 \mathrm{~dB}$ for $\left[\Gamma=10^{-4}\right.$, $\left.A=10^{-4}\right]$ ). To help network designers to choose the best parameters and/or enhance the receiver with a soft decision, the next step is to propose a theoretical performance estimation of LoRa communication in such an impulsive noise context.

\section{REFERENCES}

[1] F. Ait Aoudia, M. Gautier, M. Magno, M. Le Gentil, O. Berder, and L. Benini, "Long-Short Range Communication Network Leveraging LoRa and Wake-up Receiver," Microprocessors and Microsystems: Embedded Hardware Design (MICPRO), vol. 56, pp. 184 - 192, December 2017.

[2] C. Goursaud and J.-M. Gorce, "Dedicated networks for IoT : PHY / MAC state of the art and challenges," EAI endorsed transactions on Internet of Things, Oct. 2015.

[3] S. K. Rao and R. Prasad, "Impact of 5G technologies on industry 4.0," Wireless Personal Communications, vol. 100, no. 1, pp. 145-159, May 2018.

[4] O. Seller and N. Sornin, "Low power long range transmitter," Aug. 2014, US Patent App. 14/170,170. 
TABLE II

DiFFERENT TIMES ON AIR ACCORDING TO $S F, B F$ AND $C R$ PARAMETERS WITH A 20-BYTE PAYLOAD.

\begin{tabular}{|c|c|c|c|c|c|}
\hline$S F$ & 7 & 7 & 12 & 12 & 12 \\
\hline$B W(\mathrm{kHz})$ & 500 & 125 & 500 & 500 & 125 \\
\hline$C R$ & $4 / 5$ & $4 / 5$ & $4 / 5$ & $4 / 8$ & $4 / 8$ \\
\hline ToA $(\mathrm{ms})$ & 14.144 & 56.576 & 329.728 & 428.032 & 1712.128 \\
\hline
\end{tabular}

[5] Semtech, "SX1272/73-860 MHz to $1020 \mathrm{MHz}$ low power long range transceiver," Tech. Rep. Datasheet, Mar. 2015.

[6] Y. Roth, J.-B. Doré, L. Ros, and V. Berg, "Contender waveforms for low-power wide-area networks in a scheduled 4G OFDM framework," EURASIP Journal on Advances in Signal Processing, vol. 2018, no. 1, p. 43, Jul 2018.

[7] R. Sanchez-Iborra and M.-D. Cano, "State of the art in LP-WAN solutions for industrial IoT services," Sensors, vol. 16, no. 5, 2016.

[8] M. Rizzi, P. Ferrari, A. Flammini, E. Sisinni, and M. Gidlund, "Using LoRa for industrial wireless networks," in IEEE 13th International Workshop on Factory Communication Systems (WFCS), May 2017, pp. $1-4$.

[9] A. Guizar, L. S. Filho, M. Maman, and V. Mannoni, "Massive deployment evaluation of adaptive LPWA networks using turbo-FSK," in 14th International Conference on Wireless and Mobile Computing, Networking and Communications (WiMob), 2018, pp. 1-8.

[10] G. Madi, F. Sacuto, B. Vrigneau, B. L. Agba, Y. Pousset, R. Vauzelle, and F. Gagnon, "Impacts of impulsive noise from partial discharges on wireless systems performance: application to MIMO precoders," EURASIP Journal on Wireless Communications and Networking, vol. 2011, no. 1, p. 186, Nov 2011.

[11] B. L. Agba, F. Sacuto, M. Au, F. Labeau, and F. Gagnon, Wireless Communications for Power Substations: RF Characterization and Modeling, S. I. Publishing, Ed., 2019.

[12] D. Middleton, "Statistical-physical models of electromagnetic interference," IEEE transactions on Electromagnetic Compatibility, no. 3, pp. 106-127, 1977.

[13] H. Soury, F. Yilmaz, and M. Alouini, "Average bit error probability of binary coherent signaling over generalized fading channels subject to additive generalized Gaussian noise," IEEE Communications Letters, vol. 16, no. 6, pp. 785-788, June 2012.

[14] M. Shao and C. L. Nikias, "Signal processing with fractional lower order moments: stable processes and their applications," Proceedings of the IEEE, vol. 81, no. 7, pp. 986-1010, July 1993.

[15] T. Shongwe, A. J. H. Vinck, and H. C. Ferreira, "On impulse noise and its models," in 18th IEEE International Symposium on Power Line Communications and Its Applications, March 2014, pp. 12-17.

[16] R. Sanchez-Iborra and M. Cano, "State of the art in LP-WAN solutions for industrial IoT services," Sensors, vol. 16, no. 5, p. 708, 2016 [Online]. Available: https://doi.org/10.3390/s16050708

[17] A. Springer, W. Gugler, M. Huemer, L. Reindl, C. C. W. Ruppel, and R. Weigel, "Spread spectrum communications using chirp signals," in IEEE/AFCEA EUROCOMM, Information Systems for Enhanced Public Safety and Security, 2000, pp. 166-170.

[18] B. Reynders and S. Pollin, "Chirp spread spectrum as a modulation technique for long range communication," in SCVT, Nov. 2016, pp. 15.

[19] X.-C. Le, B. Vrigneau, M. Gautier, M. Mabon, and O. Berder, "Energy/reliability trade-off of LoRa communications over fading channels," in 25th International Conference on Telecommunications (ICT), June 2018, pp. 544-548.

[20] J. G. Proakis, Digital Communications, 5th edition, N. York:McGrawHill, Ed., 1995.

[21] T. Elshabrawy and J. Robert, "Closed form approximation of LoRa modulation BER performance," IEEE Communications Letters, vol. 22, no. 9, pp. 1778-1781, 2018.

[22] J. Courjault, B. Vrigneau, and O. Berder, "Fast performance evaluation of LoRa communications over Rayleigh fading channels," in IEEE Wireless Communications and Networking Conference (WCNC), workshop MoTION, 2019

[23] A. Mathur, M. R. Bhatnagar, and B. K. Panigrahi, "Performance evaluation of PLC under the combined effect of background and impulsive

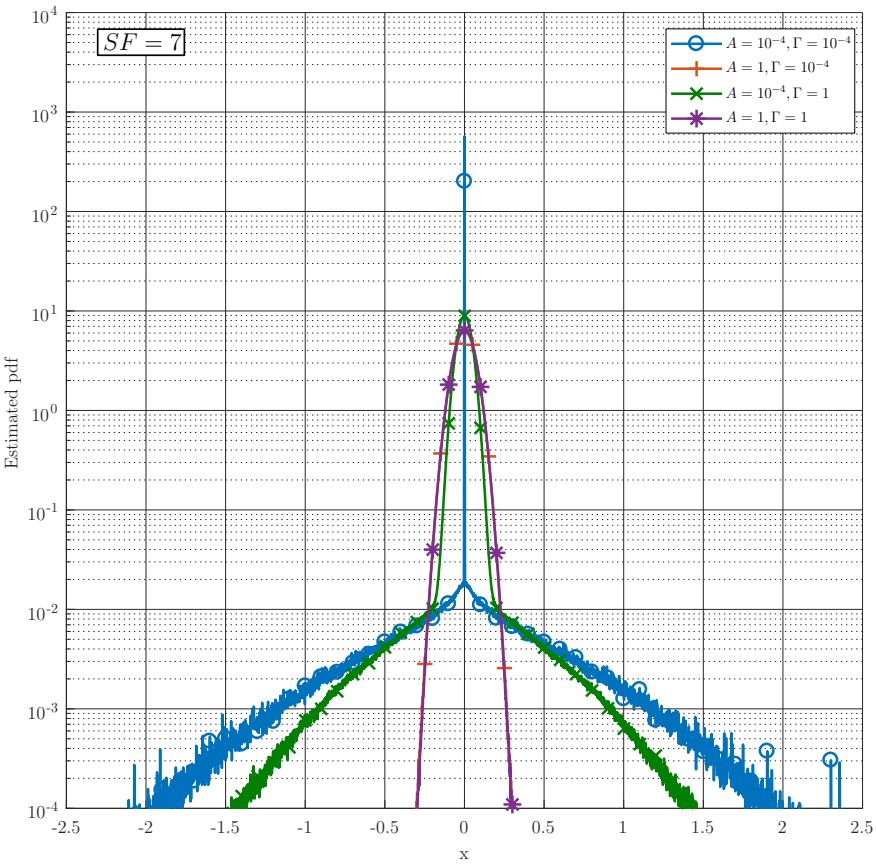

(a) Estimated PDF for $S F=7$

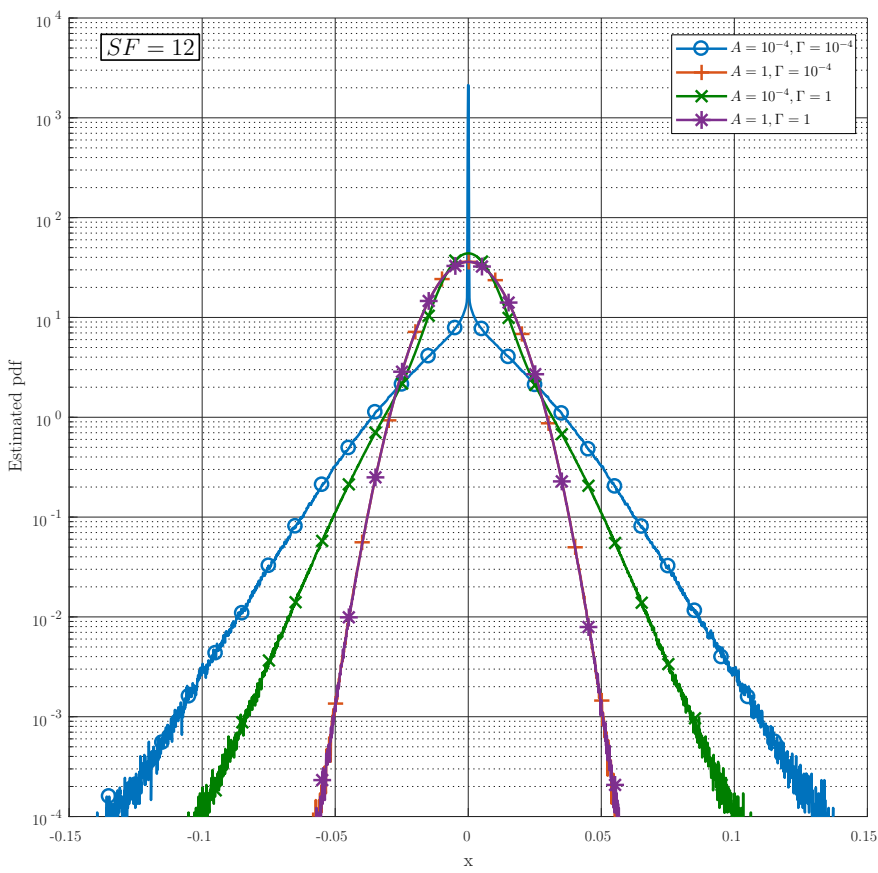

(b) Estimated PDF for $S F=12$

Fig. 9. Estimated PDF of MCAN after demodulation (real part after the FFT).

noises," IEEE Communications Letters, vol. 19, no. 7, pp. 1117-1120, July 2015.

[24] T. Elshabrawy and J. Robert, "Evaluation of the BER Performance of LoRa Communication using BICM Decoding," in IEEE 9th International Conference on Consumer Electronics (ICCE-Berlin), Sep. 2019, pp. 162-167. 